\title{
Relationship Between High Intra-Abdominal Pressure and Compliance of the Pelvic Floor Support System in Women Without Pelvic Organ Prolapse: A Finite Element Analysis
}

Xiaode Liu

Peking University

Qiguo Rong

Peking University

Jianliu Wang

Peking University People's Hospital

Bing Xie

Peking University People's Hospital

Shuang Ren ( $\square$ xixishuang123@126.com )

Peking University Third Hospital https://orcid.org/0000-0001-9644-6434

Research article

Keywords: pelvic floor support system, finite element method, prolapse, compliance, healthy woman.

Posted Date: November 15th, 2021

DOI: https://doi.org/10.21203/rs.3.rs-1075420/v1

License: (c) (i) This work is licensed under a Creative Commons Attribution 4.0 International License.

Read Full License 


\section{Abstract}

Background: The objective of this study was to study the relationship between high intra-abdominal pressure and the compliance of the pelvic floor support system in a normal woman without pelvic organ prolapse (POP), using a finite element model of the whole pelvic support system.

Methods: A healthy female volunteer (55 years old) was scanned using magnetic resonance imaging (MRI) during the Valsalva maneuver. According to the pelvic structure contours traced by a gynecologist and anatomic details measured from dynamic MRI, a finite element model of the whole pelvic support system was established, including the uterus, vagina with cavity, cardinal and uterosacral ligaments, levator ani muscle, rectum, bladder, perineal body, pelvis, and obturator internus and coccygeal muscles. This model was imported into ANSYS software, and an implicit iterative method was employed to simulate the biomechanical response with increasing intra-abdominal pressure.

Results: Stress and strain distributions of the vaginal wall showed that the posterior wall was more stable than the anterior wall under high intra-abdominal pressure. Displacement at the top of the vagina was larger than that at the bottom, especially in the anterior-posterior direction.

Conclusion: These results imply potential injury areas with high intra-abdominal pressure in nonprolapsed women, and provide insight into clinical managements for the prevention and surgical repair plans of POP.

\section{Background}

Pelvic organ prolapse (POP) is a common problem for elder women specifically for the females after postmenopause and is generally associated with defects or injuries of the pelvic floor support system [1]. Half of all parous women have experienced POP, and 10-20\% lifetime risk need surgical care[2]. Increased intra-abdominal pressure, such as with loaded walking, coughing, sneezing, squatting, defecating and bending, is an important independent risk factor for POP [3]. Constipation as well as obesity can also induce chronic high intra-abdominal pressure [2].

A number of studies [4-6] have simulated anterior and posterior vaginal wall prolapse under high intraabdominal pressures. These studies showed that combined injury to the levator ani muscle and the vaginal apex results in anterior vaginal wall prolapse, and combined injury to the levator ani muscle and posterior supporting tissues leads to posterior vaginal wall prolapse. These findings were of great significance for exploring the mechanism of vaginal prolapse. However, the studies mainly focused on the relationship between the size of the prolapse and injury to the supporting tissues, and neglected the strain and stress distributions of the supporting tissues as well as high-risk areas of injury. Another important factor for prolapse is the effect of supporting tissues on organs and the interactions between organs. Few studies have reported these two crucial factors in healthy people. 
The objective of this study was to simulate the pelvic visceral mechanical response of non-prolapsed women under high intra-abdominal pressure using a three-dimensional (3D) finite element model (FEM) of the pelvic floor support system. The model was established using ANSYS software (ANSYS, Houston, TX, USA), and the anatomy of the single volunteer subject was obtained by magnetic resonance imaging (MRI). Vaginal wall displacement and the distributions of stress and strain in the supporting tissues were calculated, and possible initial damage points in the supporting tissue and the relationship between intraabdominal pressure and pelvic floor visceral displacement were studied.

\section{Methods}

\subsection{Reconstruction of 3D FEM}

One asymptomatic and physical examination confirmed healthy female volunteer (55 years old, BMI: $20.96 \mathrm{~kg} / \mathrm{m}^{2}$ ) with no previous pelvic surgery was recruited. The subject signed informed consent for inclusion in this institutional review board-approved study. This is a 50th demographic percentile subject from a IRB-approved case-control mechanistic cohort study at the Peking University People's Hospital comparing women with anterior vaginal wall prolapse with normal asymptomatic women (Institutional Review Board HUM00012823). Axial, sagittal, and coronal MRI images were acquired while the subject was in the supine position during rest using a 3.0-T GE scanner (Discovery MR750 3.0 T; GE Healthcare, Milwaukee, WI, USA) with a 32-channel, torso phased-array coil. The pelvic structure contours were traced with the following parameters: TR/TE 3000/102-108 ms; field of view 26-28 cm; slice thickness $4 \mathrm{~mm}$ interleaved; gap $1 \mathrm{~mm}$; acquisitions 2, and 90 continuous images were obtained. The MRI images were then imported into the medical image processing software Mimics 10.01 (Materialise Inc., Leuven, Belgium) for 3D calculations and reconstruction by an experienced urogynecologist. The 3D model was segmented into anatomic structures, including pelvic bones, bladder, urethra, vagina, uterus, rectum, obturator internus, cardinal ligaments, uterosacral ligaments, and five branchese of the levator ani muscles. Then every single structure above was exported as STL files and imported into Geomagic Studio software (version 12.0; Geomagic, Inc., Morrisville, NC, USA) for more detailed pre-processing, such as smoothing and positioning. Finally, the entire model was imported into ANSYS software version 14.0 (Houston, TX, USA) for the study in this paper. The detailed description of FEM was described and validated in our previous publication [7].

\subsection{Material properties}

All material properties were considered linear elastic to simplify the numerical simulations. The elastic modulus of the level III supporting was based on perineal body data derived from in vivo measurements of healthy nulliparous women [8]. Uniaxial tension data from cadaveric specimens $[9,10]$ was used for the elastic modulus of the apex ligaments. Since there are no existing data describing the fascial properties in the literature, we assumed that the elastic modulus of the fascia was half that of the perineal body, and that the elastic modulus of the abdominal cavity was half that of the fascia. Poisson's ratio for the fascia and abdominal cavity was 0.3 and 0.49 , respectively. 
The mechanical properties of the vagina in previous studies were mostly measured in vitro, and the data varied from one study to another. Considering that the properties of connective tissue in the abdominal cavity in vivo are quite different from those in vitro, and that the vagina was similar to fascia based on the clinical experience, we chose $0.015 \mathrm{MPa}$ instead of using the data in the literature, for this study. The data for the bladder and rectum were scaled up to the same magnitude as that of the vagina according to measurements in previous studies. Regarding the uterus, few studies have reported uterine material properties in nulliparous women. Therefore, we used the characteristics of uterine samples from pregnant women for this analysis. At high intra-abdominal pressure, contraction of the muscles in the pelvic wall (including the peritoneum) leads to higher elastic modulus of tissues; thus, the pelvic wall had the same properties as the attached muscles. All of the material parameters mentioned above are presented in Table 1.

Table 1

Material parameters of pelvic tissue

\begin{tabular}{|lll|}
\hline Tissue & $\begin{array}{l}\text { Poisson ratio ( } \\
\text { Vvarvec } \mu)\end{array}$ & $\begin{array}{l}\text { Modulus of elasticity } \\
\text { (E) MPa }\end{array}$ \\
\hline levator ani muscle [11] & 0.3 & 0.8654 \\
\hline posterior ligamentous complex [9] & 0.3 & 0.3251 \\
\hline perineal body [8] & 0.3 & 0.0289 \\
\hline vagina & 0.3 & 0.015 \\
\hline uterus [12] & 0.49 & 0.486 \\
\hline bladder [13] & 0.49 & 0.01519 \\
\hline rectum [13] & 0.45 & 0.01142 \\
\hline abdominal cavity & 0.49 & 0.0072 \\
\hline fascia & 0.3 & 0.0144 \\
\hline $\begin{array}{l}\text { coccygeal muscle, obturator muscles and } \\
\text { peritoneal wall [14] }\end{array}$ & 0.3 & 13.37 \\
\hline
\end{tabular}

\subsection{Boundary conditions}

We assumed that the pelvis was fixed, and that all nodes in the pelvis were fully constrained. The ligaments and muscles could not move relative to the pelvis. Regarding the load conditions, the intraabdominal pressure during the Valsalva maneuver mentioned in the literature $[15,16]$ was approximately 70-168 $\mathrm{cm} \mathrm{H}_{2} \mathrm{O}$. In this study, we loaded with a uniform $100 \mathrm{~cm} \mathrm{H}_{2} \mathrm{O}(0.01 \mathrm{MPa})$ intra-abdominal pressure on the peritoneum to represent the situation under higher intra-abdominal pressure. When the volunteer performed the Valsalva maneuver, the abdominal wall moved $5 \mathrm{~mm}$ forward, which was documented via dynamic MRI. Thus, the abdominal wall in this model involved a 5-mm forward 
displacement. We used the pre-conditioned conjugate gradient method to perform the analysis until convergence was obtained. It took approximately 30 minutes to complete the simulation on a computer with an Intel ${ }^{\circledR}$ Core $^{\mathrm{TM}}$ i7-4790 processor (IBM Corp., Armonk, NY) with $3.60 \mathrm{GHz}$ CPU and $32.0 \mathrm{G}$ RAM running Windows 7 professional version (Microsoft Corp., Redmond, WA, USA).

\section{Results}

\subsection{Compliance}

Backward and downward displacement of the vagina were observed with increasing intra-abdominal pressure under normal pelvic support. Displacement of the top of the vagina was larger compared with that of the bottom of the vagina. The results were in good agreement with the vaginal displacement observed in dynamic MRI, as shown in Table 2.

For the anterior and posterior vaginal walls, 13 nodes were selected from the top to the bottom. The most distal edge of the cervix was set as the $C$ point, and the compliance of the anterior vaginal wall, posterior vaginal wall, and the $C$ point was explored.

Table 2

Comparison of vaginal displacement under high intra-abdominal pressure

\begin{tabular}{|lll|}
\hline & $\begin{array}{l}\text { Displacement from } \\
\text { MRI(mm) }\end{array}$ & $\begin{array}{l}\text { Displacement from } \\
\text { simulation(mm) }\end{array}$ \\
\hline $\begin{array}{l}\text { Proximal point of anterior vaginal } \\
\text { wall in y }\end{array}$ & 10.24 & 5.29 \\
\hline $\begin{array}{l}\text { Proximal point of anterior vaginal } \\
\text { wall in z }\end{array}$ & 7.81 & 8.1 \\
\hline $\begin{array}{l}\text { Distal point of anterior vaginal wall } \\
\text { in y }\end{array}$ & -2.9 & -0.3 \\
\hline $\begin{array}{l}\text { Distal point of anterior vaginal wall } \\
\text { in z }\end{array}$ & 5.9 & 5.6 \\
\hline
\end{tabular}

The vertical, and forward and backward directions were defined as the $Z$ direction and $Y$ direction in a coordinate system, respectively. The largest vertical displacement occurred at the top of the bladder, while the smallest displacement was detected at the side walls of the uterus and vagina and at the bottom of the bladder. Figure 3 shows the compliance of the anterior vaginal wall and the posterior vaginal wall, with 1-cm $\mathrm{H}_{2} \mathrm{O}$ increments of intra-abdominal pressure. Absolute values were used for analysis as all the displacements were negative (Fig. 1). Regarding the $Z$ direction, the compliance of the anterior vaginal wall (Fig. 1, in blue) showed a downward trend from the top to the bottom, which was consistent with the clinical observation. The compliance of the anterior vaginal wall was slightly higher than that of the posterior vaginal wall. In the upper third of the vagina, the compliance of the anterior and posterior vaginal walls showed the most significant difference. As the $C$ point of the cervix was supported by the 
cardinal and uterosacral ligaments complex, its downward compliance was lower than that of the vaginal wall (Fig. 1(a)). The $Y$ direction compliance of the vaginal wall decreased gradually from the top to the bottom, and its descending speed was higher than that in the $Z$ direction (Fig. 1). The $Y$ direction displacement compliance of the anterior vaginal wall was higher than the posterior vaginal wall, similar to the $\mathrm{Z}$ direction.

\subsection{Distributions of stress and strain in the supporting tissues of the pelvic floor}

On the fascia, ligaments, and muscle, the compressive strength was higher than the tensile and shear strength, and the area with highest strain was more inclined to be injured. In our study, a set of elements with high strain in the pelvic support structure were selected to calculate the maximum principal and shear strains. The maximum positive principal strain showed that the levator ani muscle bore tension, and the maximum negative principal strain indicated that the levator ani muscle underwent compression. As shown in Fig. 2(A), the levator ani muscle bore tension in the middle of the front and both sides of the back. Higher tension and shear strains were detected from the junction of the levator ani muscle and obturator internus to the junction of the anterior levator ani muscle and pubic bone. Meanwhile, strain at the junction of the levator ani muscle and coccygeal muscle was relatively high, reaching more than 0.15 .

The area with concentrated strain was detected at the upper connection between the right cardinal ligaments and the cervix, which had an amplitude of more than 0.2 (Fig. 2(B)). The upper third of the vaginal lateral wall bore high tensile strain, while the lower parts bore high shear strain. The upper anterior vaginal wall and the top of the vagina also bore high shear strain, reaching 0.5 (Fig. 2(C)). The tensile and shear strains at the junction of the pubocervical fascia and the obturator muscles were higher compared with other tissues, and the maximum shear strain in the $Y Z$ direction was more than 0.7 (Fig. 2(D)). Similar results were found at the rectovaginal fascia and the side of the mesorectum, as shown in Fig. 2(E) and Fig. 2(F), respectively.

The supporting tissue elements with high strain were selected to calculate the maximum principal strain and shear strain. The edge of the supporting tissues was not considered owing to the strain concentrations that may result from the calculation process. Figure $3(A-C)$ summarize the strain values with intra-abdominal pressures ranging from $0.002 \mathrm{MPa}$ to $0.01 \mathrm{MPa}$ for each tissue. Strain values increased with increasing intra-abdominal pressure. The maximum principal strain was detected at the pubocervical fascia Fig. 3(A), and the $Y-Z$ shear strain reached 0.9 at the top of the pubocervical fascia Fig. 3(C). A concentrated stress area was more likely at the levator ani muscle-obturator internus junction and cardinal ligaments-pelvic junction, which implied a high risk of clinical levator ani muscle and pelvic prolapse. The tension and shear strains for the lateral fascia were higher than those of the middle part connected with the organs. Thus, the probability of fascial and arcus tendineus fasciae pelvis prolapse was higher than that of a tear in the middle part connected with organs. This finding was also in agreement with the clinical result that there was a higher probability of displacement anterior wall prolapse than dilatation anterior wall prolapse. 
Figure 3(D) and Fig. 3(E) show the maximum principle and shear stresses of the pelvic support structures, respectively. A similar trend was observed in that the maximum principle stress and shear stress increased with increasing intra-abdominal pressure. The maximum principle stress was $0.041 \mathrm{MPa}$ at the top of the cardinal ligaments (Fig. 3(D)), and the maximum shear stress was $0.037 \mathrm{MPa}$ at the levator ani muscle-coccygeus junction Fig. 3(E). These results indicated that the levator ani muscle and cardinal ligaments bore high tensile stress, as the maximum stress amplitude was very close between the levator ani muscle-obturator internus junction and the top of cardinal ligaments Fig. 3(D). This was in line with clinical expectations and previous research results showing that the levator ani muscle and cardinal ligaments were the main support structures in load-bearing. The shear stress in the levator ani muscle was higher than that of the cardinal ligaments Fig. 3(E).

\section{Discussion}

In this study, the compliance of the whole pelvic floor support system in a healthy female was studied using finite element analysis based on MRI. The vaginal wall displacement and the distributions of stress and strain in the supporting tissues were calculated under high intra-abdominal pressure. Our results showed that displacements of the vagina obtained in this study were in agreement with the data measured using clinical dynamic MRI. Figure 4 shows the displacement of the anterior vaginal wall compared with Larson et al's results reconstructed from dynamic MRI at rest and during the Valsalva maneuver [17]. Our results and previous studies compare favorably. The results were also similar to those using dynamic MRI in asymptomatic volunteers (Fig. 4(b)). All of these evaluation results confirmed the effectiveness of our study.

The posterior vaginal wall was more stable than the anterior vaginal wall in non-prolapsed women under high intra-abdominal pressure. In the vertical direction, the $C$ point at the top was more stable than the anterior and posterior vaginal walls, while the stability of the $\mathrm{C}$ point was poorer in the anterior-posterior direction. According to our results, the anterior vaginal wall had the worst stability, which explained the high incidence of cystocele [18], clinically.

Previous studies showed that compliance varied in different vaginal regions [19]. Compliance was the highest at the top of the vagina and lowest at the vaginal introitus. Our results were consistent with this observation and implied that non-prolapsed women also had compliance during high intra-abdominal pressure, and that vaginal wall movement should be restored intra-operatively, rather than be completely constrained [19]. In a previous study [19], compliance in the top and bottom of the anterior vaginal wall was approximately $0.51 \mathrm{~mm} / \mathrm{cm} \mathrm{H}_{2} \mathrm{O}$ and $0.18 \mathrm{~mm} / \mathrm{cm} \mathrm{H}_{2} \mathrm{O}$, respectively. The corresponding values were lower in our study at $0.048 \mathrm{~mm} / \mathrm{cm} \mathrm{H}_{2} \mathrm{O}$ and $0.09 \mathrm{~mm} / \mathrm{cm} \mathrm{H}_{2} \mathrm{O}$, respectively. However, our results matched well with the dynamic MRI results of the volunteer during the Valsalva as well as physical examination in Peking University People's Hospital. This discrepancy could be explained by two reasons: first, healthy women may vary in vaginal compliance; second, Spahlinger et al's research [19] was based on Caucasian anatomy, and our study was based on Asian anatomy. Different perineal body shapes and vaginal lengths could also lead to different compliance results. 
Regarding strain in the vagina and supporting tissues, we found that strain at the sides of the levator ani muscle and pelvic fascia was higher, indicating that there were high risks of clinical prolapse between the levator ani muscle and arcus tendineus musculi levatoris ani and between the pelvic fascia and arcus tendineus fasciae pelvis. These areas were also susceptible to tear injuries, resulting in a higher risk of injury to the paravaginal supporting tissues. Previous studies reported that the incidence of paravaginal defects in patients with anterior vaginal wall prolapse was 38-80\% [20-22], and indicated that debonding of the pubocervical fascia and arcus tendineus fasciae pelvis clinically was the gold standard for diagnosing paravaginal defects [20]. This study showed that both the levator ani muscle and the sides of the pelvic fascia were at a high risk of injury. Our results suggest that attention should be paid to lateral vaginal repair intraoperatively, which was also confirmed in Viana et al's study [23]. The authors studied 66 women with symptomatic cystocele (grade 2-4) who underwent transvaginal paravaginal repair. Results showed that suspending the vesicovaginal fascia to the arcus tendineus fasciae pelvis was a safe and effective method for the treatment of paravaginal defects in patients with symptomatic cystocele, and the recurrence rate within 1 year was low (8.5\%). The long-term effect of traditional anterior vaginal repair is poor, with a high recurrence rate of symptomatic cystocele.

In this study, we found that there was a high risk of injury at the top of the cardinal ligaments and cervix. High strains were also detected at the vaginal sidewall and the upper anterior vaginal wall, which may be related to the broadening of the vaginal wall. The results indicated that special attention should be paid to these regions in clinical evaluations, and comprehensive repair plans should be made to avoid complications and reduce the recurrence rate as much as possible.

The levator ani muscle and the cardinal sacral ligament complex bore high tensile and shear forces, which theoretically proved that the pelvic floor plays an important role in the supporting tissues. This result was consistent with the finding by Chen et al [4]. The levator ani muscle bore high $\mathrm{Y}-\mathrm{Z}$ shear stress to prevent the pelvic viscera from excessive downward movement. According to previous studies, the levator ani muscle has fiber orientation. This muscle has a strong load-bearing capacity along the horizontal fiber direction, while its vertical fiber direction cannot bear excessive load. The distribution of fibers in the levator ani muscle was from one side of the obturator internus to the other side of the obturator internus [24], which was the $X$ direction in our model. Therefore, the levator ani muscle could not bear excessive $\mathrm{Y}-\mathrm{Z}$ shear load, and the side of the levator ani muscle and the back area connected with the coccygeal muscle was more susceptible to injury.

Our results showed that the pelvic floor support system in a healthy female was sensitive to increasing intra-abdominal pressure. Chronic high intra-abdominal pressure, such as with obesity, chronic cough, and chronic constipation are risk factors for POP $[25,26]$. Avoiding high intra-abdominal pressure exercises as well as training to increase pelvic floor muscle strength could reduce the risk of pelvic floor support tissue injury and prevent POP.

Regarding the boundary conditions in the finite element analysis, previous studies applied the load perpendicular to the vaginal sidewall to simulate the pelvic system during high intra-abdominal pressure. 
Chen et al [4] applied a perpendicular pressure to the surface of the anterior vaginal wall, and Luo et al [6] applied the pressure perpendicular to the nodes on the anterior and posterior vaginal wall, perineal body, and levator ani muscle. However, accurate loads on the vaginal wall surface are difficult to estimate [27], and the anterior vaginal wall in vivo is connected with the pubocervical fascia, not directly exposed to the abdominal cavity. Thus, the simulated perpendicular pressure on the vaginal wall surface differs from the actual conditions in vivo. Chen et al [27] introduced a new idea in displacement loading by applying a specific displacement on the top of the uterus to simulate intra-abdominal pressure. However, applying displacement constraint only on the top of the uterus leads to inaccurate stress distribution in other organs that are not loaded because intra-abdominal pressure is transmitted to the surface of organs through the peritoneum in vivo. In the current study, we first established a peritoneal structure, and then applied uniform intra-abdominal pressure on its surface. The pressure was transmitted to the surface of the pelvic organs through the connective tissues, which more closely simulated natural pelvic loading.

There are limitations should be acknowledged. First, although the isotropic linear elastic material parameters were derived from previous studies, the material properties may vary with different methods and different measurement conditions. Furthermore, some tissues, such as the perineal body, ligamentous complex, and fascia, exhibited viscoelastic properties; however, we did not consider the effect of a load that changes over time. Second, this study focused only on the passive stretching of the levator ani muscle, as the intra-abdominal pressure was applied by the volunteer under the condition of levator ani muscle relaxation. Anisotropy, hyperelasticity, and active contractility of the levator ani muscle were not taken into account.

\section{Conclusions}

Based on the FEM reconstructed from the dynamic MRI, this study simulated the compliance of the whole pelvic floor support system of non-prolapsed women under high intra-abdominal pressure. The results of this study were in good agreement with clinical data and previous data, which confirmed the validity of this finite element analysis. Our results showed that the posterior vaginal wall of non-prolapsed women was more stable than the anterior vaginal wall under high intra-abdominal pressure. The compliance at the top of the vagina was larger than that at the bottom of the vagina, especially in the anterior-posterior direction. The stability of the $\mathrm{C}$ point in the vertical direction was higher than that of the vaginal wall, while the stability was worse in the anterior-posterior direction.

In conclusion, this is the first study to investigate the distributions of strain and stress as well as the highrisk injury areas in the whole pelvic floor support system. Based on the biomechanical characteristics of healthy women, our results showed that the levator ani muscle, the sidewall of the pelvic fascia, proximal of the cardinal ligaments, vaginal sidewall, and the upper anterior vaginal wall were vulnerable to injury due to the development of stress concentration. These findings can be used to evaluate the potential injury areas in non-prolapsed women under high intra-abdominal pressure. We also suggest that comprehensive clinical repair plans should be made to reduce post-operative complications and recurrence rates. 


\section{Abbreviations}

POP

pelvic organ prolapse; MRI:magnetic resonance imaging; 3D:three-dimensional; FEM:finite element model.

\section{Declarations}

\section{Ethics approval and consent to participate}

Study approval was granted by the Ethics Committee at Peking University People's Hospital (Reference Number IRB00001052-18018).The healthy female volunteer has given written informed consent to data collection, analysis and the use of these data for research.

\section{Consent for publication}

Written consent for the publication of identifying images and/or data in this manuscript was provided by the participant.

\section{Availability of data and materials}

The datasets used and/or analysed during the current study are available from the corresponding author on reasonable request.

\section{Competing interests}

All authors declare that they have no conflict of interest.

\section{Funding}

We would like to gratefully acknowledge the financial support of Natural Science Foundation of China Grant (31900943), Peking University Medicine Seed Fund for Interdisciplinary Research, the Fundamental Research Funds for the Central Universities (BMU20140410), UMHS-PUHSC Joint Institute for Translational and Clinical Research (BMU2008JI005) Peking University People's Hospital Scientific Research Development Funds (RDM2019-08), China Postdoctoral Science Foundation Grant (2018M631279).

\section{Authors' contributions}

QR and SR contributed to the conception of the study.JW, XL and BX performed the clinical experiment. SR contributed significantly to finite element analysis and data analyses. $X L$ performed the manuscript preparation and wrote the manuscript. QR, SR and XL helped perform the analysis with constructive discussions.

\section{Acknowledgment}


We thank Jane Charbonneau, DVM, from Liwen Bianji, Edanz Group China (http://www.liwenbianji.cn/ac), for editing the English text of a draft of this manuscript.

\section{Author details}

${ }^{1}$ Department of Mechanics and Engineering Science, College of Engineering, Peking University, 100871 Beijing, China. ${ }^{2}$ Department of Sports Medicine, Peking University Third Hospital, Institute of Sports Medicine of Peking University, Beijing Key Laboratory of Sports Injuries, 100191 Beijing, China. ${ }^{3}$ Department of Obstetrics and Gynecology, Peking University People's Hospital, Peking University, Beijing 100044, China. ${ }^{4}$ Beijing Key Laboratory of Female Pelvic Floor Disorders, The Research Center of Female Pelvic Floor Disorder Disease of Peking University, Beijing 100044, China. ${ }^{5}$ Joint International Research Center of Translational and Clinical Research, Beijing 100191, China

\section{References}

1. Tillack AA, Joe BN, Yeh BM, Jun SL, Kornak J, Zhao S, Deng D. Vaginal shape at resting pelvic MRI: predictor of pelvic floor weakness? Clinical imaging. 2015;39(2):285-8.

2. Thakar R, Stanton S. Management of genital prolapse. BMJ: British Medical Journal. 2002;324(7348):1258-62.

3. Indumathi K, Majeroni BA, Johnson DW. \%J American Family Physician: Pelvic organ prolapse. 2010, 81(9):1111-1117.

4. Chen L, Ashton-Miller JA, DeLancey JOL. A 3D finite element model of anterior vaginal wall support to evaluate mechanisms underlying cystocele formation. J Biomech. 2009;42(10):1371-7.

5. Chen L, Ashton-Miller JA, Hsu Y, DeLancey JOLJO. Gynecology: Interaction Among Apical Support, Levator Ani Impairment, and Anterior Vaginal Wall Prolapse. 2006, 108(2):324-332.

6. Luo J, Chen L, Fenner DE, Ashton-Miller JA, DeLancey JOL. A multi-compartment 3-D finite element model of rectocele and its interaction with cystocele. J Biomech. 2015;48(9):1580-6.

7. Ren S, Xie B, Wang J, Rong Q. Three-dimensional modeling of the pelvic floor support systems of subjects with and without pelvic organ prolapse. Biomed Res Int. 2015;2015:845985.

8. Chen L, Low LK, DeLancey JO, Ashton-Miller JA. In vivo estimation of perineal body properties using ultrasound quasistatic elastography in nulliparous women. J Biomech. 2015;48(9):1575-9.

9. Venugopala Rao G, Rubod C, Brieu M, Bhatnagar N, Cosson M. Experiments and finite element modelling for the study of prolapse in the pelvic floor system. Comput Methods Biomech Biomed Engin. 2010;13(3):349-57.

10. Yan X, Kruger JA, Nielsen PMF, Nash MP. Effects of fetal head shape variation on the second stage of labour. J Biomech. 2015;48(9):1593-9.

11. Nagle AS, Barker MA, Kleeman SD, Haridas B, Douglas Mast T. Passive biomechanical properties of human cadaveric levator ani muscle at low strains. J Biomech. 2014;47(2):583-6. 
12. Manoogian SJ, Bisplinghoff JA, Kemper AR, Duma SM. Dynamic material properties of the pregnant human uterus. J Biomech. 2012;45(9):1724-7.

13. Rubod C, Brieu M, Cosson M, Rivaux G, Clay J-C, de Landsheere L, Gabriel B. Biomechanical properties of human pelvic organs. Urology. 2012;79(4):968. e917-968. e922.

14. Jing D: Experimental and Theoretical Biomechanical Analyses of the Second Stage of Labor. Dissertation. Michigan: University of Michigan; 2010.

15. Chen L, Ashton-Miller JA, DeLancey JO. A 3D finite element model of anterior vaginal wall support to evaluate mechanisms underlying cystocele formation. J Biomech. 2009;42(10):1371-7.

16. Chen L, Ashton-Miller JA, Hsu Y, DeLancey JO. Interaction among apical support, levator ani impairment, and anterior vaginal wall prolapse. Obstet Gynecol. 2006;108(2):324-32.

17. Larson KA, Hsu Y, Chen L, Ashton-Miller JA, DeLancey JOL. Magnetic resonance imaging-based three-dimensional model of anterior vaginal wall position at rest and maximal strain in women with and without prolapse. Int Urogynecol J. 2010;21(9):1103-9.

18. Hendrix SL, Clark A, Nygaard I, Aragaki A, Barnabei V. Obstetrics AMJAJo, Gynecology: Pelvic organ prolapse in the women\"s health initiative: Gravity and gravidity. 2002, 186(6):1160-1166.

19. Spahlinger DM, Newcomb L, Ashton-Miller JA, DeLancey JOL, Chen LJIUJ. Relationship between intra-abdominal pressure and vaginal wall movements during Valsalva in women with and without pelvic organ prolapse: technique development and early observations. 2014, 25(7):873-881.

20. Segal JL, Vassallo BJ, Kleeman SD, Silva WA, Journal MMKJIU. Paravaginal defects: prevalence and accuracy of preoperative detection. 2004, 15(6):378-383.

21. Richardson AC, Lyon JB. Williams NLJAJoO, Gynecology: A new look at pelvic relaxation. 1976, 126(5):568-573.

22. Obstetrics YOUNGBLOOD. JPJC, Gynecology: Paravaginal Repair for Cystourethrocele. 1993, 36(4):960-966.

23. Viana R, Colaço J, Vieira Á, Gonçalves V, Retto HJIUJ. Cystocele-vaginal approach to repairing paravaginal fascial defects. 2006, 17(6):621-623.

24. Shobeiri SA, Chesson RR, Gasser RF: The internal innervation and morphology of the human female levator ani muscle. American Journal of Obstetrics and Gynecology 2008, 199(6):686.e681686.e686.

25. O'Dell KK, Morse AN. It's Not All About Birth: Biomechanics Applied to Pelvic Organ Prolapse Prevention. Journal of Midwifery Women's Health. 2008;53(1):28-36.

26. Bump RC, Norton PA. EPIDEMIOLOGY AND NATURAL HISTORY OF PELVIC FLOOR DYSFUNCTION. Obstet Gynecol Clin North Am. 1998;25(4):723-46.

27. Chen Z-W, Joli P, Feng Z-Q, Rahim M, Pirró N, Bellemare M-E. Female patient-specific finite element modeling of pelvic organ prolapse (POP). J Biomech. 2015;48(2):238-45.

\section{Figures}



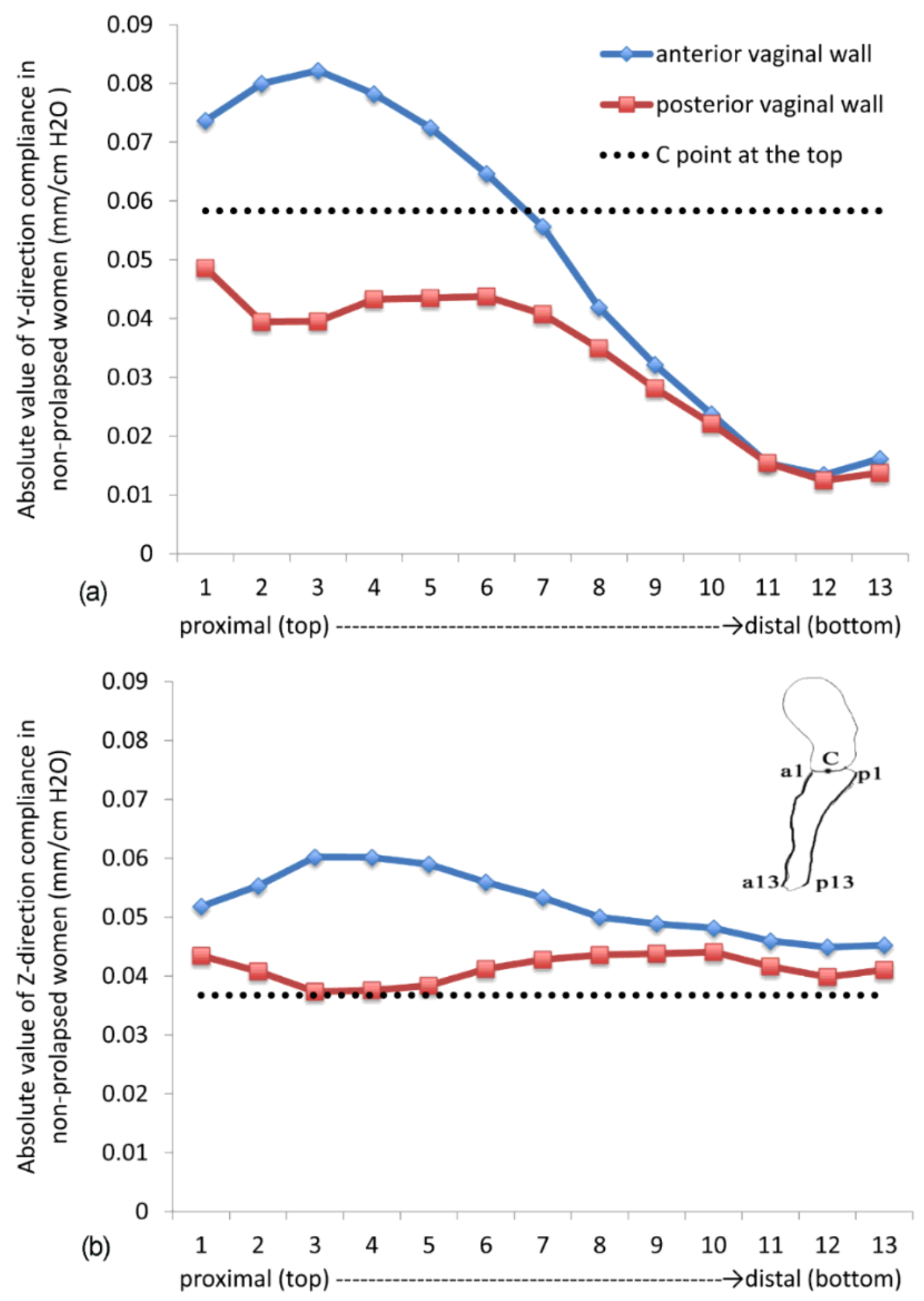

\section{Figure 1}

Absolute values in the (a) (forward and backward) Y direction and (b) (vertical) Z direction compliances in non-prolapsed women. 
Levator ani muscle

(A)

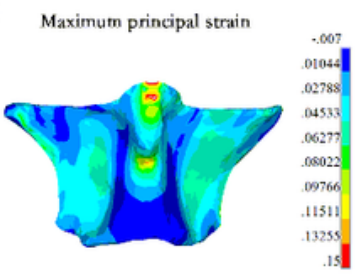

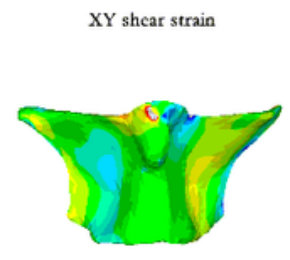

.15
.016666
.05333
.05
.01666
.01600
.05
.08333
.11666
.15

(B)
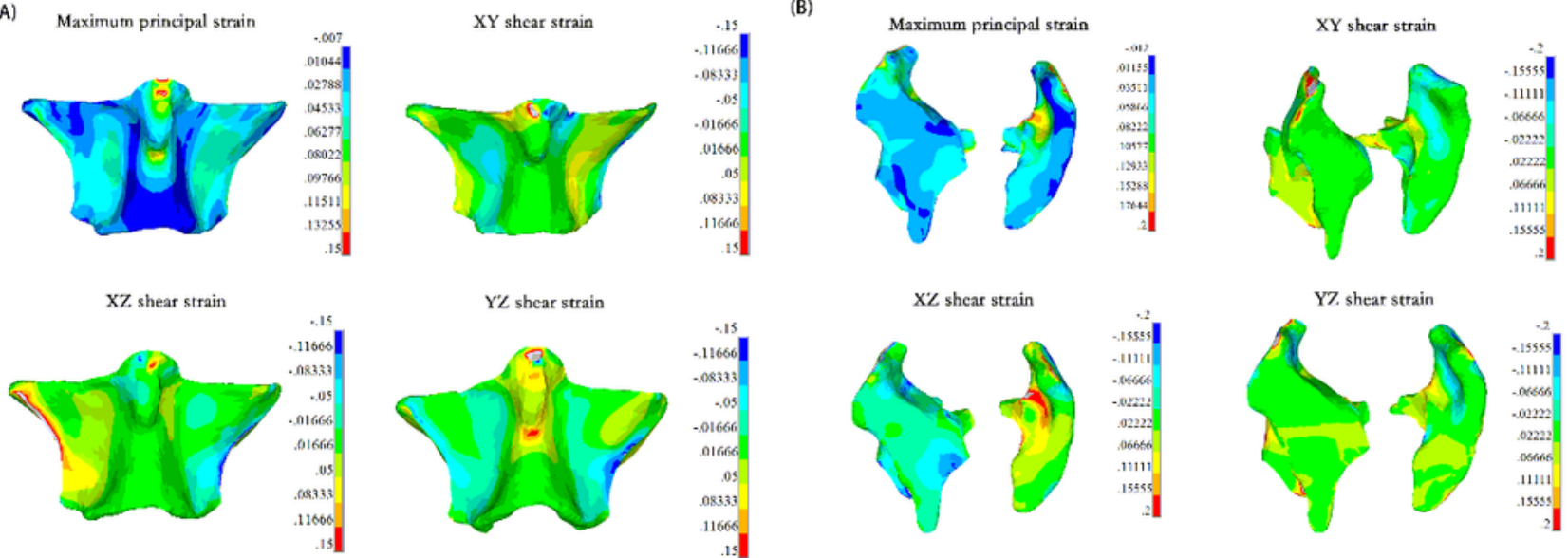

.15
-11666
-0.03333
.05
-0.0666
.016666
.05
.08333
.11666
.15
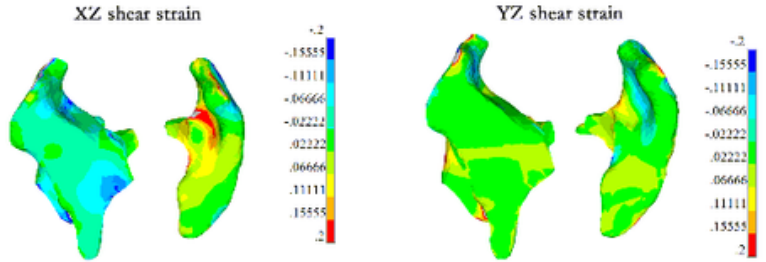

Pubocervical fascia
(C)

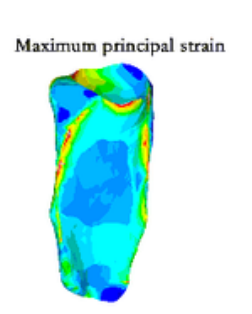

$\mathrm{X} Z$ shcar strain

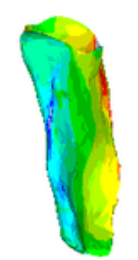

Vagina

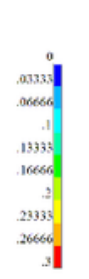

$$
\begin{array}{r}
-5 \\
.38898 \\
.2777 \\
.16666 \\
.05555 \\
.05555 \\
.16666 \\
.2777 \\
.38888 \\
.5
\end{array}
$$

Rectovaginal fascia

(E)
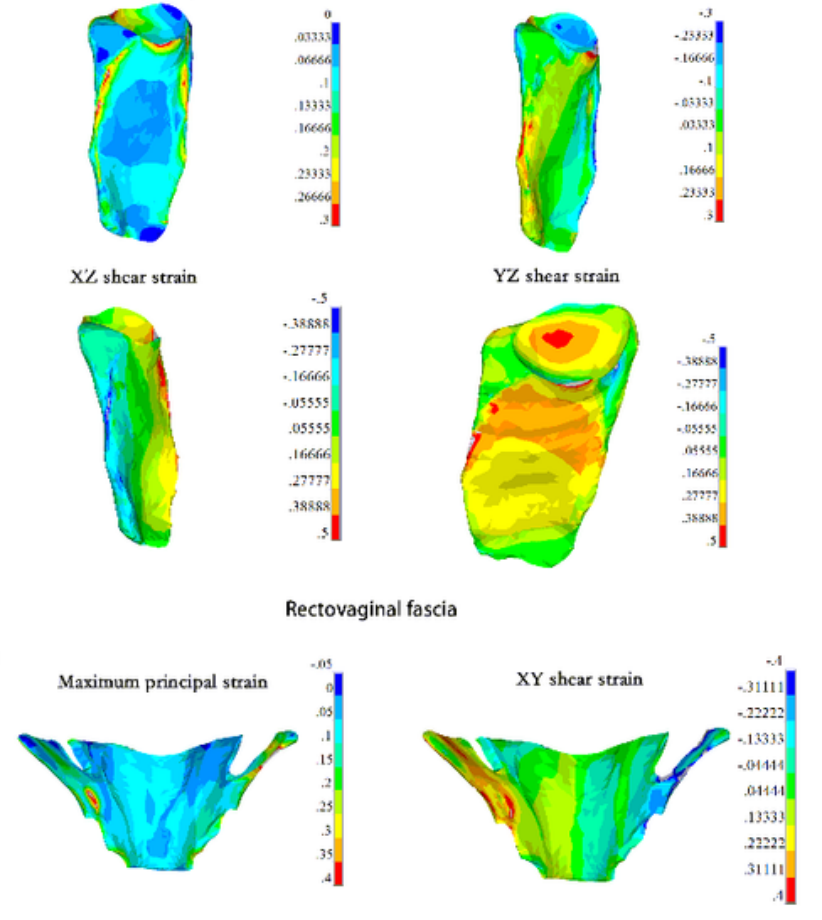

(D)
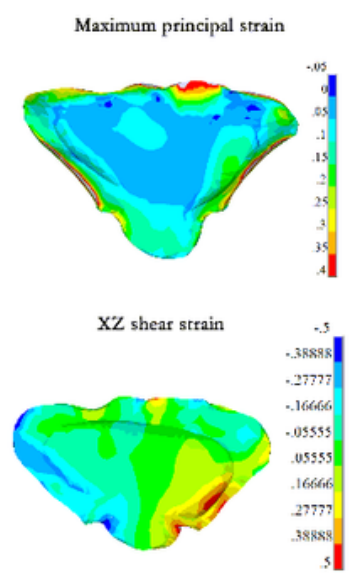

Mesorectum

(F)
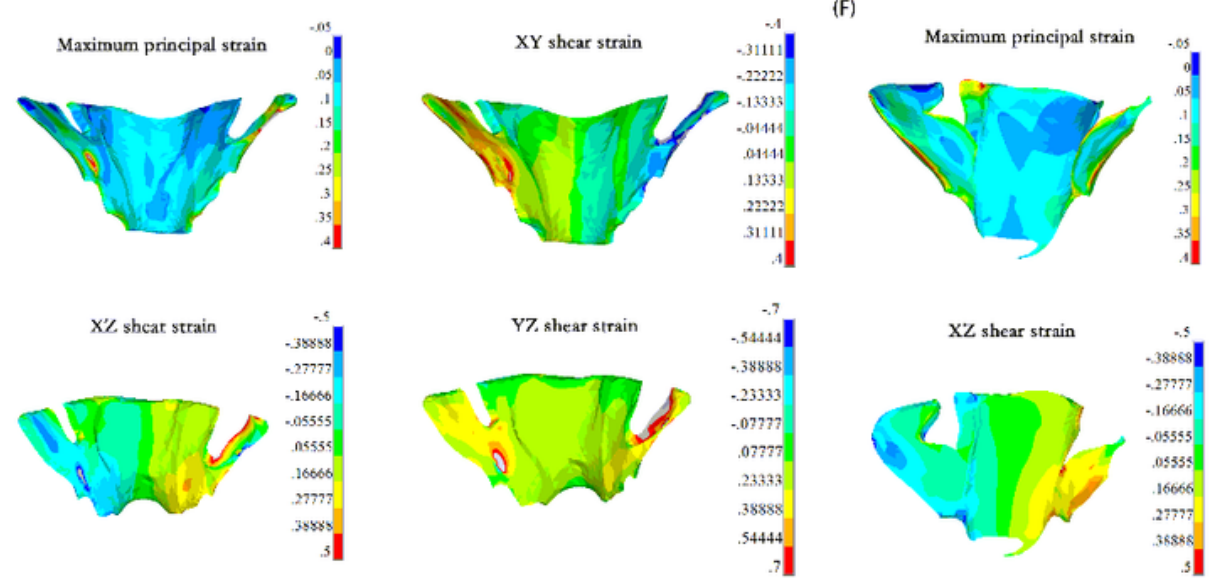
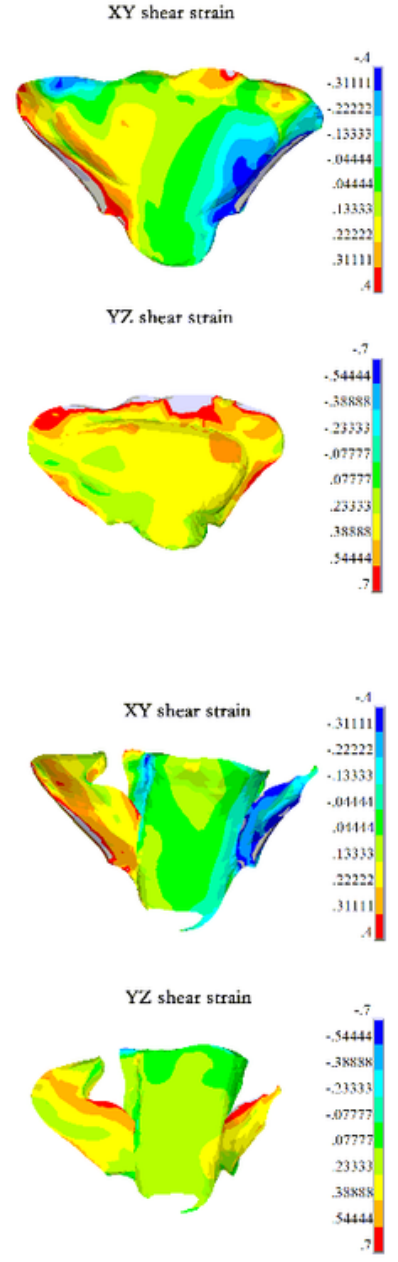

Figure 2

Strain in the pelvic organs under $0.01 \mathrm{MPa}$ intra-abdominal pressure 
Strain

(A)

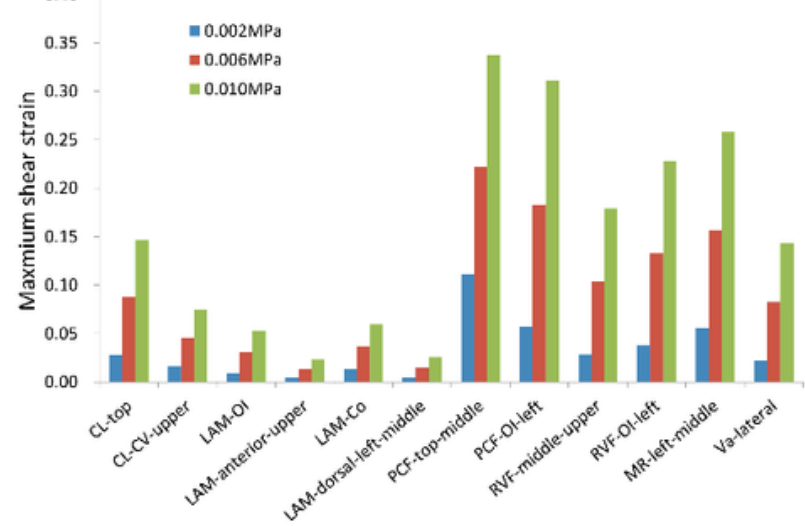

(B)

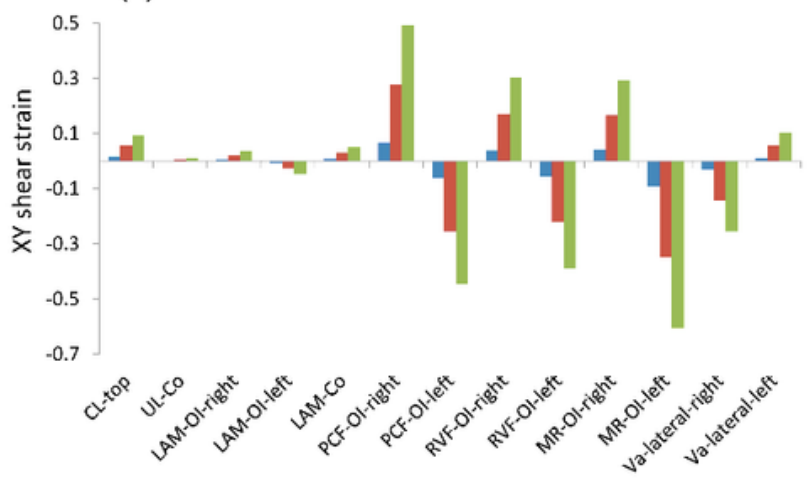

(C)

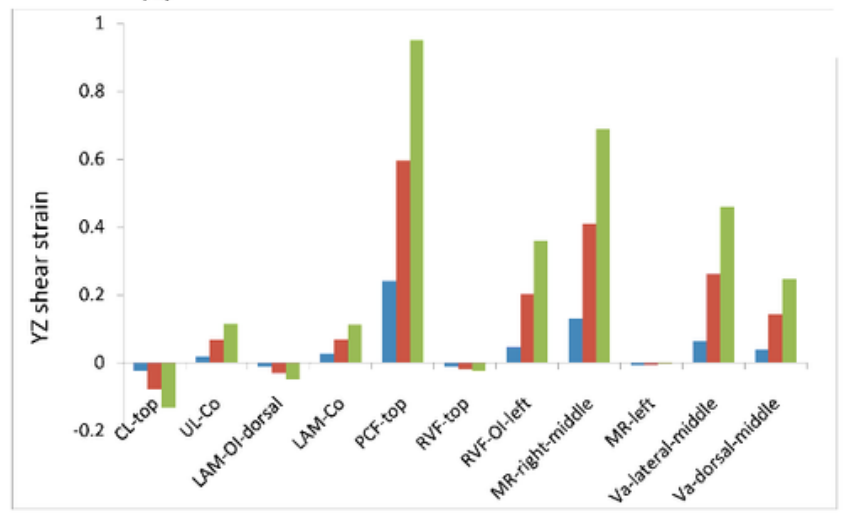

(D)
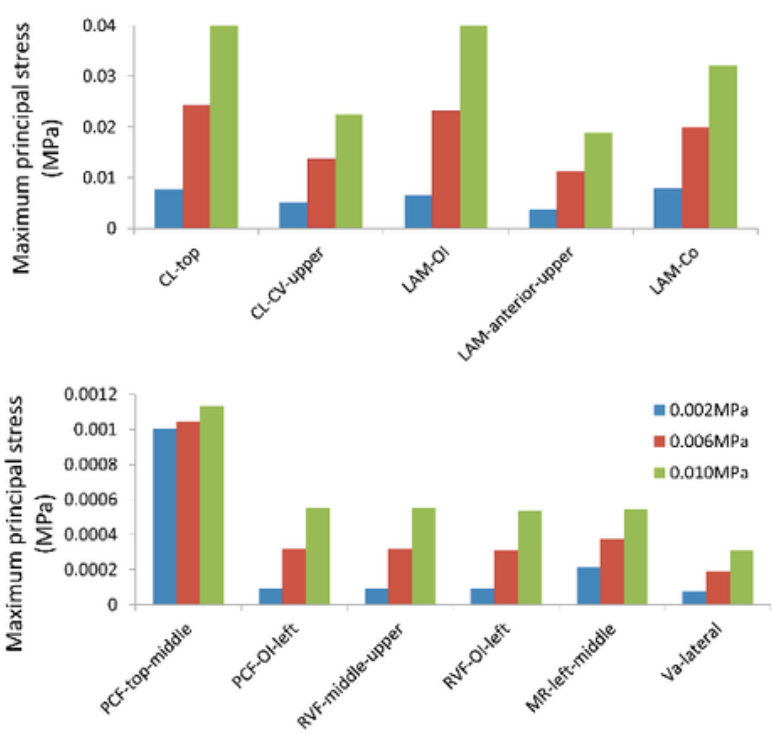

(E)
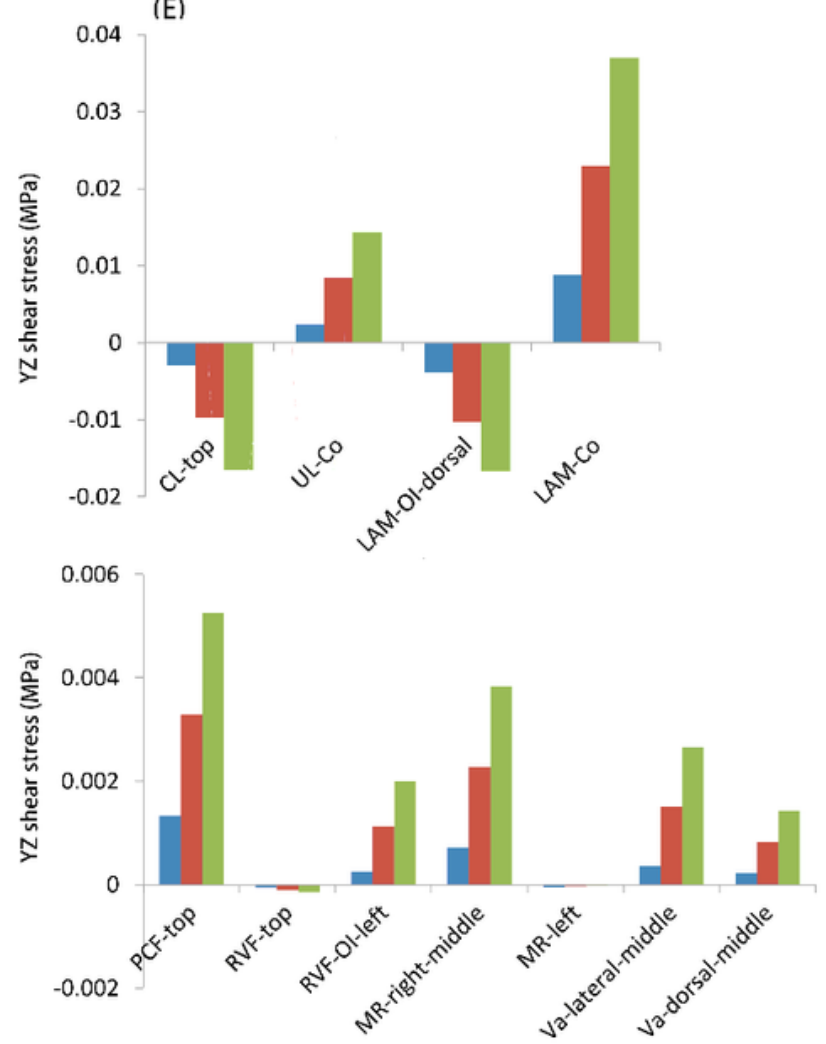

Figure 3

Stress and strain in the supporting tissues. CL: cardinal ligaments; CL-CV: cardinal ligaments-cervical junction; LAM: levator ani muscle; LAM-OI: levator ani muscle-obturator internus junction; LAM-Co: levator ani muscle-coccygeus junction; PCF: pubocervical fascia; PCF-OI: pubocervical fascia-obturator internus junction; RVF: rectovaginal fascia; RVF-OI: rectovaginal fascia-obturator internus junction; MR: 
mesorectum; MR-OI: mesorectum-obturator internus junction; UL-Co: uterosacral ligaments-coccygeus junction; Va: vagina.

\section{Resting}

(a)

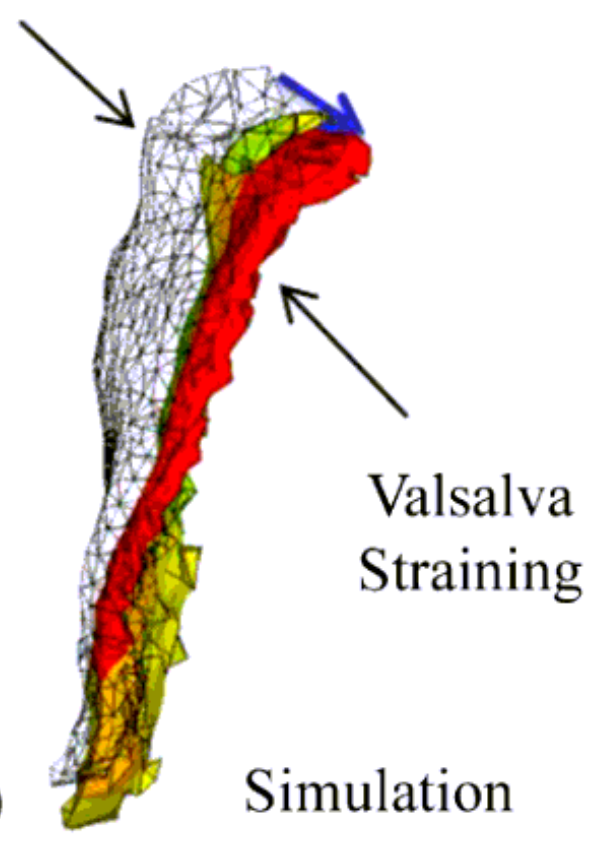

Resting

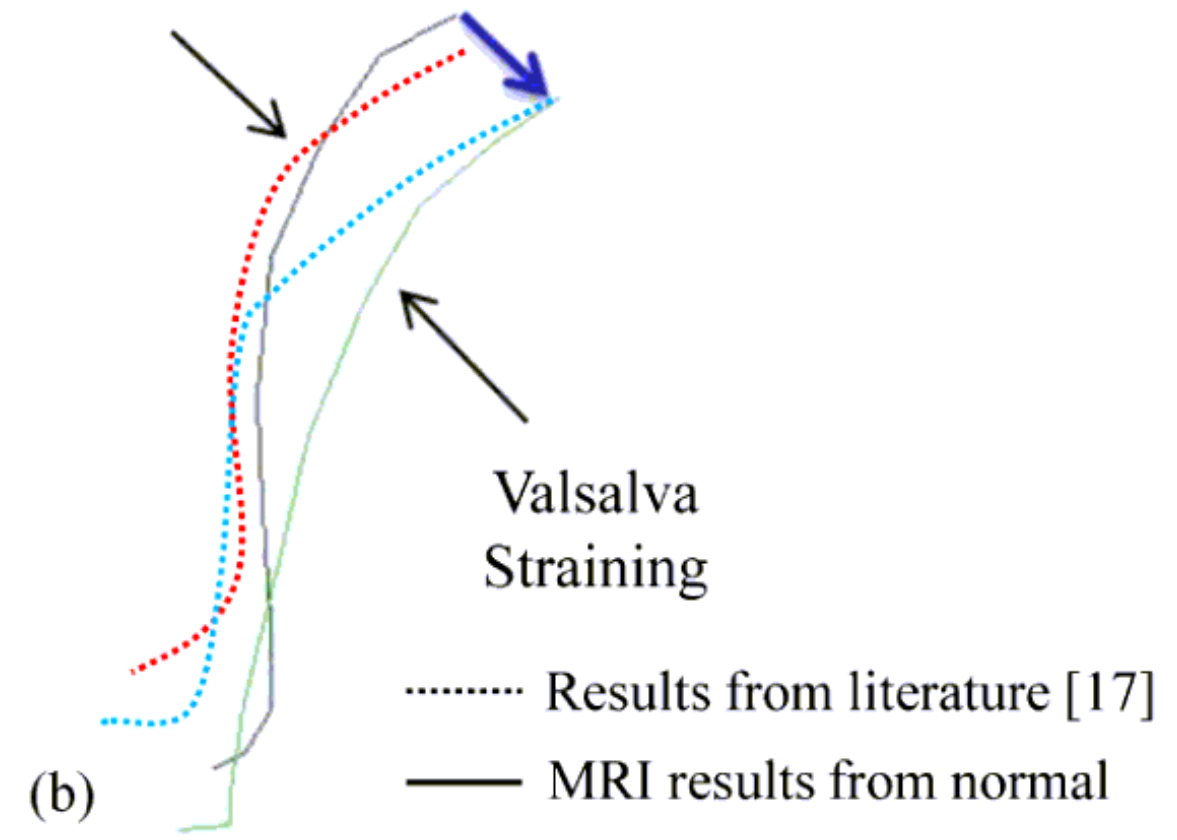

\section{Figure 4}

Displacement of the anterior vaginal wall during the Valsalva maneuver: (a) Results from our simulation; (b) Results from a previous study [17] and dynamic MRI. 